\title{
A QUALIDADE DE VIDA DA PESSOA COM DEFICIÊNCIA VISUAL
}

\author{
Olivete Oliveira ${ }^{1}$ \\ Célia Ribeiro ${ }^{2}$ \\ Cristina Simões ${ }^{3}$
}

\begin{abstract}
Resumo: Atualmente vivemos numa sociedade com predominante apelo ao uso da visão. A visão é um elemento extremamente determinante na relação com os objetos, com o espaço $e$, naturalmente, com os outros. Na sua ausência ou perante uma grande perturbação, uma afetação negativa na capacidade visual de uma pessoa, que não se consegue melhorar, mesmo com correção ótica e recurso a meios convencionais, estamos na presença de uma deficiência visual (DV). Sabendo-se que a DV é uma problemática que limita a vida das pessoas, a mesma pode afetar a sua qualidade de vida $(Q V)$.

A $Q V$ é um conceito que tem vindo a ser alvo de interesse no âmbito de várias áreas do saber, sendo que a sua definição varia de acordo com as mesmas, não existindo uma definição única $e$ generalizável. Contudo, pode-se afirmar que a QV é um conceito multidimensional e hierárquico, que inclui medidas subjetivas $e$ objetivas. Este constructo tem assumido um papel muito importante nos cuidados de saúde, nos serviços sociais, nas famílias $e$ naturalmente na área da Educação/Educação Especial, bem como para o desenvolvimento de políticas nacionais e internacionais destinadas às pessoas com deficiência e na avaliação dos resultados pessoais.
\end{abstract}

Palavras-chave: Deficiência Visual, Baixa Visão, Cegueira, Qualidade de Vida.

\footnotetext{
${ }^{1}$ Mestre em Ciências da Educação, Especialização em Educação Especial. Docente de Educação Especial. E-mail: olivete_oliveira@hotmail.com

2 Doutorada em Psicologia, Área de Especialização em Psicologia Pedagógica. Professora Auxiliar da Universidade Católica Portuguesa - Viseu. E-mail: cribeiro@viseu.ucp.pt

3 Doutorada em Educação Especial. Docente de Educação Especial e Professora Assistente convidada da Universidade Católica Portuguesa. E-mail: cristinasimoes.qv@gmail.com
} 


\title{
Title: QUALITY OF LIFE OF PEOPLE WITH VISUAL IMPAIRMENT
}

\begin{abstract}
We currently live in a society with a predominant appeal to the use of vision. Vision is an extremely decisive factor in relation to objects, with space and naturally with others. In his absence or before a large disturbance, a negative affectation in the visual ability of a person, that you can not improve, even with optical correction and use of conventional means, we have a visual impairment (VI). Knowing that the VI is a problem that limits the lives of people, of course that it can affect his/her quality of life ( $Q O L)$.

Quality of life is a concept that has been the target of interest within various areas of knowledge, and its definition varies according to the same, thus there is no single and generalizable definition. However, it can be said that $Q O L$ is a multidimensional and hierarchical concept that includes subjective and objective measures. This concept has played a very important role in health care, social services, families and of course in Education / Special Education as well as to the development of national and international policies for people with disabilities and the evaluation of the personal outcomes.
\end{abstract}

Keywords: Visual Impairment, Low Vision, Blindness, Quality of Life.

\section{INTRODUÇÃO}

A Qualidade de Vida (QV) de cada um está presente nos modos de vida social, na cultura, ou seja, na estrutura social, e ter mais ou menos QV é algo que se expressa diariamente, nos pequenos momentos da vida quotidiana de cada sujeito, de forma única e específica (Veiga, Silva, Domingues, Saragoça, \& Fernandes, 2014).

Ao longo da história, o conceito de QV tem sido alvo de grande interesse por parte de diversas áreas, tais como a sociologia, a economia, a política, a psicologia, a saúde e a educação, que atribuem ao termo múltiplos significados relacionados com o conhecimento e os valores individuais ou coletivos divergentes em diversas épocas, locais e histórias (Goode, 1994; Raphael, 1996; Raphael, Brown, \& Renwick, 1996). Contudo, estas diferentes abordagens e conceitos, bem como o facto de a QV possuir significados pessoais distintos, levam a avaliações e resultados das pesquisas científicas diferenciados.

A QV tem vindo a ganhar uma crescente importância, tem-se tornado fulcral para o fornecimento de informações relevantes para a avaliação de resultados, a reabilitação, a definição de estratégias e práticas dos 
serviços, assim como para a criação e orientação de políticas orientadas para as pessoas com deficiência.

Dado que a Deficiência Visual (DV) tem consequências no dia a dia das pessoas, interferindo na sua autonomia e independência, é do nosso interesse efetuar uma revisão da literatura sobre os principais estudos que relacionam a QV e a DV e destacar conclusões que nos permitam ter um melhor conhecimento sobre a temática.

\section{O CONCEITO DE QUALIDADE DE VIDA}

O conceito de QV não é novo (Claes, Van Hove, Van Loon, Vandevelde, \& Schalock, 2010; Schalock et al., 2002). É um conceito abrangente e variado, baseando-se em recursos da ciência e da arte, manifestando-se não só na pesquisa de dados e na sua medição, mas também numa variedade de expressões pessoais como o bem-estar individual e a pertença (Brown et al., 2000; Schalock et al., 2002). Compreendê-lo assume uma particular importância para as práticas educativas atuais. Analisando a literatura, a QV é definida de diferentes maneiras, desde um requerimento genérico para a felicidade e as experiências de vida consideradas positivas (Claes et al., 2010).

$\mathrm{Na}$ consideração de Farquhar (1995) e Pereira, Teixeira e Santos (2012), a variedade de definições reflete as perspetivas e as orientações profissionais dos investigadores, sendo que a existência de distintas conceções e perspetivas teóricas sobre a QV dificultam a operacionalização do termo. Esta constatação levou Dantas, Sawada e Malerbo (2003) e Seild e Zanonn (2004) a considerarem a QV como uma temática de difícil compreensão e que necessita de certas delimitações com vista a facilitar a sua operacionalização em análises científicas. É por isso que Pereira, Teixeira e Santos (2012) consideram que o desenvolvimento de uma classificação ou taxonomia de QV seja útil, não somente para demonstrar a falta de consensos, mas, também, para organizar as definições existentes e, desse modo, identificar e ordenar os elementos comuns e os fatores de destaque que influenciam estas definições.

No sentido de clarificar o conceito, um grupo de especialistas da Organização Mundial de Saúde (OMS; 1994) apresentou uma definição de QV, que mais tarde foi partilhada por Skevington, Lotfy e O'Connell (2004) e segundo os quais a QV é "a percepção do indivíduo sobre a sua posição na vida, dentro do contexto dos sistemas de cultura e valores nos quais está inserido e em relação aos seus objectivos, expectativas, padrões 
e preocupações" (OMS, 1994, p. 28). É portanto, uma definição que equaciona o conceito de QV enquanto produto da relação do sistema pessoal do indivíduo com os seus contextos de vida e com o ambiente sociocultural (Centro de Reabilitação Profissional de Gaia, 2007; Guggenmoos-Holzmann, Bloomfield, Brenner, \& Flick, 1995; Hegarty, 1994; OMS, 1994, 1998; Piteira, 2000) e que deve ser vista como uma experiência pessoal singular, uma vez que, cada pessoa experimenta as mesmas situações de forma diferente e única (Hegarty, 1994). É um conceito alargado e influenciado de forma complexa pela saúde física, estado psicológico, nível de independência, relações sociais, crenças pessoais, bem como pelas relações com o respetivo meio (OMS, 1995).

Na mesma linha de pensamento, a OMS (1998) refere que o conceito traduz a avaliação do indivíduo, contextualizada no meio físico, cultural e social onde vive, e que tem subjacentes três aspetos elementares: a subjetividade, a multidimensionalidade e a presença de dimensões positivas e negativas. Deste modo, apresenta-se como "uma visão multidisciplinar que se afasta da tradicional associação do conceito de qualidade de vida aos aspectos relacionados com o contexto de saúde" (Serra et al., 2006, p. 42).

Keith e Schalock (2000) consideram que o conceito de QV reflete o estado psicológico das pessoas, que é multicultural e universal. Cummins (2005) e Leal (2008) defendem a concetualização da QV como um constructo multidimensional e influenciada por fatores pessoais, ambientais e pela sua interação; tem componentes semelhantes para todas as pessoas; apresenta componentes objetivos e subjetivos; e é influenciada pela autodeterminação, pelos recursos, pelo sentido da vida e pela perceção de pertença. Phillips (2006) também salienta a satisfação das necessidades básicas e sociais, ao mesmo tempo que engloba a independência para tomar as suas próprias decisões sobre a sua própria vida, assumindo o seu papel de cidadão ativo e participante e usufruindo das mesmas condições e princípios que os seus pares. Leal (2008, pp. 1819) expressa a QV como sendo

o resultado da soma do meio ambiente físico, social, cultural, espiritual e económico onde o indivíduo está inserido, dos estilos de vida que este adopta, das suas acções e da sua reflexão sobre si mesmo, sobre os outros e sobre o meio ambiente que o rodeia. É também a soma das expectativas positivas em relação ao futuro.

Na perspetiva de Birren e Dieckman (1991), Dias e Santos (2006), Piteira (2000), Ribeiro (1994), Ruta, Garratt e Leng (1994) e Shin e Jonhson (1978), o conceito de QV apresenta um caráter subjetivo, por 
estar dependente das experiências de vida de cada indivíduo e das características pessoais. Nesse sentido, Shin e Jonhson (1978) consideram que a QV consiste "na posse dos recursos necessários para a satisfação de necessidades individuais e desejos, a participação em actividades que permitem o desenvolvimento pessoal e a autorrealização, e a comparação satisfatória entre o próprio e os outros" (p. 479).

Na mesma linha de pensamento, Schalock, Gardner e Bradley (2007) consideram que existem três conceitos básicos a ter em conta e que são: os sentimentos de bem-estar geral, as oportunidades para revelar potencialidades e os sentimentos de envolvimento social positivo, definindo QV como um conceito que reflete as condições de vida desejadas pela pessoa.

Ellwein (2001), Moreno e Ximénez (1996), Ribeiro (1994) e Rosser (1997) têm salientado a importância do domínio da saúde no estudo da $\mathrm{QV}$, por ser aquele que melhor parece explicar a variação da $\mathrm{QV}$ e que está em íntima relação com os restantes domínios. A promoção da saúde é, aliás, tratada como sinónimo da promoção da QV (Ribeiro, 1997).

Assim, um conceito que surge associado ao de QV em contextos clínicos e de saúde é o de QV Relacionada com a Saúde (QVRS; Ribeiro, 1994). Para Rosser (1997) o conceito de QVRS desenvolve-se à medida que se verifica uma crescente preocupação com o acesso cada vez mais facilitado aos cuidados de saúde e supõe que as alterações na QV das pessoas possam ser atribuídas separadamente aos cuidados de saúde, em relação a outras atividades planeadas para aumentar essa qualidade.

Estudos realizados ao longo das últimas décadas permitiram definir quatro linhas orientadoras a ter em conta quando se fala de QV (Brown et al., 2000; Cummins, 2005; Schalock et al., 2002; Schalock, Gardner, \& Bradley, 2007; Schalock \& Verdugo, 2002; Verdugo, Schalock, Keith, \& Stancliffe, 2005):

- A QV é multidimensional, ou seja, é constituída por vários domínios que se podem agrupar em oito categorias: relações interpessoais, inclusão social, desenvolvimento pessoal, bem-estar físico, autodeterminação, bem-estar material, bem-estar emocional e direitos;

- Devem desenvolver-se indicadores para cada um dos domínios da $\mathrm{QV}$, tendo em consideração que estes indicadores são definidos como a relação entre a perceção, os comportamentos e as condições que revelam o bem-estar do sujeito;

- Devem estabelecer-se os aspetos objetivos e subjetivos da QV, sendo, no entanto, comum que exista pouca relação entre estes;

- Deve focar-se na previsão dos resultados de qualidade. 
Em suma, apesar de existirem diversas definições de QV não existe uma que seja amplamente aceite. No entanto, é cada vez mais claro que esta não inclui apenas fatores relacionados com a saúde, com o bem-estar físico, funcional, emocional e intelectual, mas também outros aspetos importantes da vida das pessoas, tais como o trabalho, a família, os amigos, e os contextos diários, tendo sempre como foco de atenção as perceções pessoais de quem está a ser avaliado (Gill \& Feinstein, 1994).

\section{A QUALIDADE DE VIDA E A DEFICIÊNCIA VISUAL}

Atendendo a que ter QV “para as pessoas com deficiência significa ter vida independente, ou seja, ter controlo sobre as suas vidas, aceder às mesmas oportunidades e enfrentar as mesmas escolhas na vida diária, tal como as pessoas sem deficiência" (Veiga et al., 2014, p. 19), contribui para que na área das ciências sociais e da saúde, existam muitas disciplinas e campos com um crescente interesse em avaliar em que medida os programas e os serviços melhoram a QV destas pessoas (Verdugo et al., 2005).

As várias patologias que caracterizam a DV podem afetar negativamente a QV de um indivíduo dado que limitam as oportunidades e a autonomia na aprendizagem (Bittencourt \& Hoehne, 2006; Verdugo et al., 2005). Como resultado, a medição e promoção da QV nas pessoas que beneficiam de programas de reabilitação e de serviços a nível educacional, social e de saúde tornam-se uma prioridade (Verdugo, Caballo, Peláez, \& Prieto, 2000; Verdugo et al., 2005).

Como indica Legge (1990), as pessoas com DV podem ter dificuldades na realização de determinadas atividades diárias, como ler, conduzir, fazer uma caminhada, ver televisão, praticar desporto ou podem ter dificuldades na sua participação social. Para Alves e Duarte (2005, citados por Meereis et al., 2011), a diminuição da interação com o meio e com as pessoas que os rodeiam representa uma das grandes limitações da DV. Legge (1990) refere que a DV pode, num sentido mais amplo, ter efeitos a longo prazo de várias formas sobre a QV de um indivíduo, como por exemplo: em relação ao acesso à educação, à informação, à formação profissional, à mobilidade e à independência. Neste sentido, torna-se importante medir o impacto das limitações visuais na $\mathrm{QV}$ dessas pessoas (Verdugo et al., 2000). Contudo, as pesquisas sobre a QV das pessoas com DV são escassas. Ferguson, Buxton e Ferris (1990) constataram que, na literatura clínica relativa ao tratamento de pessoas com DV, a maioria das intervenções se restringe a medidas do sucesso de indicadores 
tradicionais, como a nitidez e o campo de visão. Os mesmos autores recomendam que, ao lado dos indicadores clínicos tradicionais, sejam consideradas mais medidas globais da $\mathrm{QV}$, no sentido de se estabelecerem relações entre a QV e os diferentes parâmetros visuais. Embora haja pouca pesquisa publicada, existe uma grande preocupação neste campo, especialmente em relação à medição da QV (Verdugo et al., 2000).

Drummond (1990) descreve a preocupação de muitos especialistas no que respeita à conceção e adaptação de instrumentos eficientes e capazes de avaliar a QV das pessoas com DV, sublinhando a necessidade de desenvolver e validar escalas, a fim de comparar a QV das pessoas com problemas visuais com indivíduos que não apresentam essas problemáticas.

Algumas pesquisas têm-se concentrado em relacionar a QV das pessoas com DV a outros conceitos, como o emprego e o estilo de vida (DeLaGarza \& Erin, 1993), a perceção da saúde (Alonso, Prieto, Ruigómez, \& Antó, 1993) e o impacto dos serviços de educação (Giangreco, Cloninger, Mueller, Yuan, \& Ashworth, 1991). Outras investigações centram-se na medição da satisfação com a vida (Davis, Lovie-Kitchin, \& Thompson, 1995). As estratégias de medição utilizadas têm sido de natureza quantitativa, com diferentes tipos de questionários a serem utilizados para medir a QV, com exceção de Giangreco e colaboradores (1991), que utilizaram uma metodologia qualitativa.

Juntamente com a escassez de estudos disponíveis, os instrumentos adaptados ou validados para a avaliação da QV das pessoas com DV são escassos e deve-se ter em consideração que estes instrumentos devem ter propriedades psicométricas adequadas, que permitam aos investigadores e profissionais avaliar os serviços e os programas (Verdugo et al., 2000).

Ellwein (2001) refere alguns instrumentos de QV relacionados com a saúde e associados especificamente à visão: o Visual Functional Questionnaire (Steinberg et al., 1994), a Activities of Daily Vision Scale (Mangione et al., 1992) e o Vision Activities Questionnaire (Sloane, Ball, Owsley, Bruni, \& Roenker, 1992). Para Ellwein (2001), o problema que se coloca quando se quer comparar resultados da QV reside precisamente no facto de serem muito diversos os instrumentos de medida, as amostras utilizadas e as variáveis consideradas. Foi neste sentido que, segundo o mesmo autor, numa tentativa de unificação das medidas de QV relacionadas com a visão, o National Eye Institute, com o apoio e colaboração de outras instituições, procurou desenvolver o Visual 
Function Questionnaire (Mangione et al., 1998). Como explica Ellwein (2001, p. 147)

o resultado deste esforço é um questionário de 25-itens, o NEI-VFQ-25, que leva aproximadamente 10 minutos para administrar. As sub escalas do VFQ incluem visão (visão geral, actividades de perto, actividades de longe, visão periférica, e visão de cor) e qualidade de vida específica da visão (funcionamento social, saúde mental, dependência, dificuldades de papel, condução, dor ocular, e saúde geral).

\subsection{Estudos}

Nos últimos anos os estudos sobre a QV têm abrangido um amplo leque de realidades, sobretudo no campo da saúde, do envelhecimento e da deficiência (Veiga et al., 2014). Através dos mesmos a QV tem sido vista como uma importante meta na avaliação dos resultados da reabilitação em geral (Renwick \& Brown, 1996) e da reabilitação das pessoas com DV em particular (Verdugo et al., 2000).

De entre os estudos relacionados com a DV destacamos os realizados por Giangreco e colaboradores (1991), Alonso e colaboradores (1993), Verdugo e colaboradores (2000), Bittencourt e Hoehne (2006), Langelaan e colaboradores (2007), Holbrook, Caputo, Perry, Fuller e Morgan (2009) e Messa, Nakanami e Lopes (2012).

Giangreco e colaboradores (1991) destacaram nas suas conclusões a importância de um lar estável, seguro e confortável, a necessidade de desenvolver uma atividade produtiva e socialmente valorizada, de cuidados de saúde e o desenvolvimento de redes e de laços sociais como indicadores de QV para as pessoas com cegueira (CG) e surdez.

A investigação levada a cabo por Alonso e colaboradores (1993) foi pensada para estudar a QV e a saúde percebida numa amostra de pessoas com CG associados à Organización Nacional de Ciegos Españoles da cidade de Barcelona e para comparar o seu estado de saúde e a sua capacidade funcional com um grupo de controlo da população geral da mesma cidade. Foram questionados trezentos e sete sujeitos e utilizados três instrumentos de medida: o Perfil de Saúde de Nottingham, o Questionário das Atividades da Vida Diária, bem como perguntas gerais sobre a saúde e os serviços de saúde.

O estudo permitiu concluir que a CG está associada a um nível de saúde percebida moderadamente baixo (fundamentalmente nas áreas da energia, sono, mobilidade física e reações emocionais) e a uma notável limitação na capacidade funcional para realizar as atividades da vida 
diária. A capacidade funcional e a saúde percebida estão mais afetadas nas populações mais idosas, nas mulheres e naqueles sujeitos que têm perceções de luz e vultos. A CG está associada a uma alta prevalência de doenças ou condições crónicas, tais como a artrose ou reumatismo, a diabetes e a surdez. Comparativamente à população em geral, verifica-se que no grupo dos sujeitos com CG, os indicadores com pior resultado são os relacionados com o estado de saúde, sobretudo na capacidade funcional, a mobilidade física e o nível de energia, e ainda a inclusão social. Estes apresentam maiores problemas de isolamento social e os problemas na limitação da sua autonomia surgem como os mais relevantes. Os sujeitos com CG utilizam mais os serviços de saúde, em virtude de um pior estado de saúde e de outras doenças associadas.

Outra investigação importante na área da QV na CG foi a de Verdugo e colaboradores (2000). Este estudo, patrocinado pela Organización Nacional de Ciegos Españoles, incidiu sobre a comunidade de Castela e Leão e foi composto por seis partes: Estudo I - Adaptação do questionário de QV de Schalock e Keith (1993, citados por Verdugo et al., 2000); Estudo II - Avaliação da QV em pessoas com DV; Estudo III - Análise de diferenças na QV entre pessoas com DV e pessoas adultas sem incapacidade; Estudo IV - Adaptação do questionário de QV do estudante de Keith e Schalock (1995, citados por Verdugo et al., 2000); Estudo V Avaliação da QV de estudantes com DV; Estudo VI - Análise de diferenças na QV entre estudantes com DV e estudantes sem incapacidade.

A amostra do estudo foi composta por quatrocentas e quarenta e oito pessoas adultas e quinhentos e trinta e um estudantes sem qualquer problemática, e por trezentas e sessenta e quatro pessoas adultas e oitenta estudantes com DV. Os investigadores utilizaram cinco instrumentos: o Quality of Life Questionnaire, o Quality of Student Life Questionnaire, o WHOQOL-Brief Questionnaire, a Escala de Nottingham e um questionário, elaborado pela equipa de investigação, que recolhia informação sobre variáveis sociodemográficas que se supunham influenciar a QV.

Desta investigação concluiu-se que o processo de adaptação dos questionários originais terminou com a construção de dois novos questionários, que apresentam diferentes subescalas e itens. $\mathrm{O}$ questionário da QV passou a ser constituído por três escalas: satisfação, competência e autodeterminação. O questionário de QV para estudantes engloba três escalas: satisfação, autodeterminação e pertença social. Ambos apresentam propriedades psicométricas adequadas para a 
população espanhola com e sem DV, pelo que poderão ser de interesse e utilidade nos campos da saúde, serviços sociais, educativos e laborais.

Ao compararem pessoas com e sem DV, os supracitados investigadores observaram que existem diferenças significativas na perceção de QV. Nos adultos, esta perceção varia em função do género, idade, residência, estado civil, situação laboral, formação, saber ler e escrever, rendimentos económicos, estado de saúde e utilização de medicação continuada. Foram encontradas diferenças significativas, a favor da população em geral, nas escalas de satisfação e autodeterminação, ainda que na variável relativa à competência as pessoas com DV com emprego sejam as que obtêm melhores pontuações. Nos estudantes com DV existem diferenças significativas em função da idade, domicílio, nível educativo e situação económica. Os estudantes com DV encontram-se mais satisfeitos comparativamente aos seus pares sem esta problemática, mas com níveis de autodeterminação mais baixos, não existindo diferenças quanto à pertença social. Finalmente, os autores salientam que as pessoas que apresentam outras deficiências associadas à $\mathrm{DV}$, as mulheres e as pessoas com mais de cinquenta anos são subgrupos das pessoas com DV que requerem uma atenção específica para melhorar a sua $\mathrm{QV}$, através do estudo das suas necessidades e problemas, com recurso a estudos qualitativos, sendo necessário o desenvolvimento de políticas e de programas que equiparem as suas oportunidades.

O estudo realizado por Bittencourt e Hoehne (2006) procurou apurar a QV numa amostra de dezasseis adultos com DV que frequentavam um programa de reabilitação de um serviço de uma Universidade no Brasil. A amostra era composta por dez sujeitos com baixa visão e seis com CG, sendo oito sujeitos do género feminino. Os resultados apontaram para uma boa ou muito boa perceção da QV por parte de 68\% dos sujeitos, principalmente ao nível do bem-estar psicológico e do bem-estar físico. Não foram encontradas diferenças estatisticamente significativas entre os sujeitos com CG e os sujeitos com baixa visão, mesmo quando se analisou a variável género (Bittencourt \& Hoehne, 2006).

Por outro lado, o estudo de Langelaan e colaboradores (2007) procedeu a uma descrição genérica da QV relacionada com a saúde e o nível de saúde de pessoas com DV, bem como a comparação da QV relacionada com a saúde com os resultados normativos da população geral da Holanda e com pessoas com problemas crónicos. Foi utilizada uma amostra de cento e vinte e oito sujeitos adultos com DV, sendo a maioria dos participantes do género feminino. Dos resultados deste estudo, destaca-se um impacto tendencialmente negativo da DV na QV. 
Quando comparada com outras doenças crónicas, os resultados obtidos indicam que a DV proporciona um maior impacto negativo na $\mathrm{QV}$ do que a diabetes tipo II, a síndrome coronária e a deficiência auditiva, e um menor impacto do que a esclerose múltipla, a síndrome de fadiga crónica ou a perturbação depressiva major (Langelaan et al., 2007).

Por sua vez, o estudo elaborado por Holbrook e colaboradores (2009) pretendeu determinar os níveis de atividade física e perceção da QV numa amostra de vinte e cinco adultos com DV, maioritariamente do género masculino. Das análises realizadas não foram encontradas diferenças estatisticamente significativas em relação à interação entre o grau de severidade da DV e a QV percebida. Por outro lado, os resultados indicaram uma tendência para os valores de QV obtidos por mulheres serem relativamente mais baixos do que os homens (Holbrook et al., 2009).

Os autores Messa, Nakanami e Lopes (2012) realizaram um estudo com o objetivo de analisar a QV de crianças com DV atendidas em Ambulatório de Estimulação Visual Precoce da Universidade Federal de São Paulo, antes e após as intervenções de reabilitação pela equipa multiprofissional. Como metodologia aplicaram o questionário Quality of Life in Visual Impaired Children Treated for Early Visual Stimulation. O instrumento tem uma versão para crianças menores de três anos e outra para crianças maiores de três anos (três a sete anos) e é dividido em seis domínios: saúde geral; saúde geral da visão; competência; personalidade e impacto familiar; e tratamento. Em síntese verificaram que a correlação entre as subescalas nos dois momentos se mostrou significativa. Houve diferenças estatisticamente significativa, relativas à saúde geral da visão ( $p=.029)$ e outras diferenças importantes foram obtidas na saúde geral, no impacto familiar e na QV geral. O instrumento mostrou-se adequado para aferir a QV percecionada pelas famílias das crianças que frequentavam esse ambulatório. Os autores concluíram que as intervenções multidisciplinares promoveram uma melhoria da visão funcional das crianças atendidas no ambulatório e da QV das famílias como um todo.

Para além dos estudos apresentados, consideramos importantes os resultados de outras investigações que associam a idade com o aumento da incapacidade funcional (Hebert, Brayne, \& Spiegelhalter, 1997). Outras investigações têm demonstrado diferenças significativas na prevalência de incapacidade funcional em função do género e, dentro destes, são as raparigas o grupo com um maior risco (Leveille, Penninx, 
Melzer, Izmirlian, \& Gurainik, 2000; Murtagh \& Hurbert, 2004; Wray \& Blaum, 2001).

Por último, destacamos os resultados do estudo realizado por Gaspar e Matos (2008), que teve como objetivo avaliar a QVRS em crianças e adolescentes e que foi aplicado a uma amostra nacional e representativa de alunos do quinto e do sétimo anos de escolaridade, num total de três mil, cento e noventa e cinco crianças e adolescentes, com idades compreendidas entre os dez e os dezasseis anos, e respetivos pais. Como resultados, as autoras destacam as diferenças significativas entre crianças e adolescentes (os mais elevados são os das crianças), em função do género (mais elevado no género masculino), do estatuto socioeconómico, da nacionalidade e do (in)sucesso escolar. Concluíram, também, que existem diferenças entre o modo como os pais avaliam os seus filhos e o modo como estes se autoavaliam.

Não obstante, dos projetos e investigações realizadas, ainda há um longo caminho a percorrer para compreender a $\mathrm{QV}$ das pessoas com DV em geral e, em particular, das crianças e adolescentes.

\section{CONCLUSÃO}

A QV é um conceito tão rico, tão vasto e tão multidimensional que defini-lo, de forma única, se tornou uma tarefa difícil, não só porque resulta da influência da área de estudo, mas também da atribuição de valores individuais ou coletivos que, naturalmente, divergem de acordo com o local e período histórico (Goode, 1994; Raphael, 1996; Raphael, Brown, \& Renwick, 1996).

Assim, ao conhecerem as perceções sobre as dimensões da QV, as famílias ficam com informações sobre como melhorar o dia a dia dos seus filhos. Deste conhecimento acrescem também informações para a escola, ao nível da educação em geral e, em particular, na Educação Especial, bem como para a prestação de serviços, nomeadamente ao nível da saúde, tal como é defendido por Brown, Schalock e Brown (2009), Keith e Schalock (2000), Rapley (2003), Schalock (1996, 2004), Schalock, Gardner e Bradley (2007) e Verdugo e colaboradores (2005).

Neste contexto, faz todo o sentido que a perceção da QV seja entendida como um conceito sensibilizante e relevante na determinação de políticas públicas, na avaliação dos serviços, e no desenvolvimento de programas inovadores a nível local, tal como o exposto por Brown e colaboradores (2000), Schalock (1996) e Schalock e Verdugo (2002). 


\section{BIBLIOGRAFIA}

Alonso, J., Prieto, L., Ruigómez, A., \& Antó J. (1993). Medida de la calidad de vida y de la salud percibida en una muestra de ciegos de Barcelona. Barcelona: Instituto Municipal d’Investigación Medica.

Birren, J., \& Dieckman, L. (1991). Concepts and content of quality of life in the later years: An overview. In J. Birren, J. Rowe, J. Lubben, \& D. Deutchman (Eds.), Quality of life in the frail elderly (pp. 344-360). New York: Academic Press.

Bittencourt, Z., \& Hoehne, E. (2006). Qualidade de vida de deficientes visuais. Revista Medicina, 39(2), 260-264.

Brown, I., Brown, R., Cummins, R., Felce, D., Matikka, L., Keith, K., Parmenter, T., \& Schalock, R. (2000). Quality of life: Its conceptualization, measurement, and application. Hastings, Nebraska: International Association for the Scientific Study of Intellectual Disabilities.

Brown, I., Schalock, R., \& Brown, R. (2009). Quality of life: Its application to persons with intellectual disabilities and their families: Introduction and overview. Journal of Policy and Practice in Intellectual Disabilities, 6(1), 2-6.

Centro de Reabilitação Profissional de Gaia (2007). Modelização das políticas e das práticas de inclusão social das pessoas com deficiência em Portugal. Vila Nova de Gaia: Centro de Reabilitação Profissional de Gaia e Instituto Superior de Ciências do Trabalho e da Empresa.

Claes, C., Van Hove, G., Van Loon, J., Vandevelde, S., \& Schalock, R. (2010). Quality of life measurement in the field of intellectual disabilities: Eight principles for assessing quality of life-related personal outcomes. Social Indicators Research, 98, 61-72.

Cummins, R. (2005). Moving from the quality of life concept to a theory. Journal of Intellectual Disability Research, 49, 699-706.

Dantas, R., Sawada, N., \& Malerbo, M. (2003). Pesquisas sobre qualidade de vida: Revisão da produção científica das universidades públicas do estado de São Paulo. Revista Latino-Americana de Enfermagem, 11(4), 532-538.

Davis, C., Lovie-Kitchin, J., \& Thompson, B. (1995). Psychosocial adjustment to age related macular degeneration. Journal of Visual Impairment and Blindness, 89, 16-27.

DeLaGarza, D., \& Erin, J. (1993). Employment status and quality of life of graduates of a state residential school. Journal of Visual Impairment and Blindness, 87, 229-233. 
Dias, J., \& Santos, S. (2006). Comportamento adaptativo e qualidade de vida: Emprego protegido na deficiência mental. Revista de Educação Especial e Reabilitação, 13, 47-58.

Ellwein, L. (2001). Quality-of-life outcome measures. In R. Massof \& L. Lidoff (Eds.), Issues in low vision rehabilitation, service delivery, policy, and funding (pp. 143-158). New York: American Foundation for the Blind Press.

Farquhar, M. (1995). Definitions of quality of life: A taxonomy. Journal of Advanced Nursing, 22(3), 502-508.

Ferguson, B., Buxton, M., \& Ferris, F. (1990). Measuring and valuating health states relating to visual impairment: A review of literature, concepts, and methods. In M. Drummond (Ed.). Measuring the quality of life of people with visual impairment: Proceedings of a workshop (pp. 6982). United States: Department of Health and Human Services.

Gaspar, T., \& Matos, M. (2008). Qualidade de vida em crianças e adolescentes: Versão portuguesa dos instrumentos Kidscreen 52. Cruz Quebrada: Aventura Social e Saúde.

Giangreco, M., Cloninger, C., Mueller, P., Yuan, S., \& Ashworth, S. (1991). Perspectives of parents whose children have dual sensory impairments. The Journal of the Association for Persons with Severe Handicaps, 16, 14-24.

Gill, T., \& Feinstein, A. (1994). A critical appraisal of the quality of qualityof-life measurements. Journal of the American Medical Association, 272(8), 619-626.

Goode, D. (1994). Quality of life of persons with disabilities: International perspectives and issues. Cambridge: Brookline.

Guggenmoos-Holzmann, I., Bloomfield, K., Brenner, H., \& Flick, U. (1995). Quality of life and health: Concepts, methods and applications. Berlin: Blackwell-Wissenschafts.

Hebert, R., Brayne, C., \& Spiegelhalter, D. (1997). Incidence of functional decline and improvement in a community-dwelling, very elderly population. American Journal of Epidemiology, 145, 935-944.

Hegarty, S. (1994). Integration and the teacher. In C. Meyer, S. Pijl, \& S. Hegarty (Eds.), New perspectives in special education: A six country study of integration (pp. 125-131). London: New York Routledge.

Holbrook, E., Caputo, J., Perry, T., Fuller, D., \& Morgan, D. (2009). Physical activity, body composition, and perceived quality of life of adults with visual impairments. Journal of Visual Impairment and Blindness, 103(1), 17-29. 
Keith, K., \& Schalock, R. (2000). Cross-cultural perspectives on quality of life. Washington, DC: American Association on Mental Retardation.

Langelaan, M., de Boer, M., Van Nispen, R., Wouters, B., Moll, A., Van Rens, G., et al. (2007). Impact of visual impairment on quality of life: A comparison with quality of life in the general population and with other chronic conditions. Ophthalmic Epidemiology, 14(3), 119-126.

Leal, C. (2008). Reavaliar o conceito de qualidade de vida. Açores: Universidade dos Açores.

Legge, G. (1990). How visual impairment affects quality of life. In M. Drummond (Ed.), Measuring the quality of life of people with visual impairment: Proceedings of a workshop (pp. 17-22). United States: Department of Health and Human Services.

Leveille, S., Penninx, B., Melzer, D., Izmirlian, G., \& Gurainik, J. (2000). Sex differences in the prevalence of morbidity disability in old age: The dynamics of incidence, recovery, and mortality. Journals of Gerontology Series B: Psychological Sciences and Social Sciences, 55, 41-50.

Mangione, C., Berry, S., Spritzer, K., Janz, N., Klein, R., Owsley, C., \& Lee, P. (1998). Identifying the content area for the 51-item National Eye Institute Visual Function Questionnaire: Results from focus groups with visually impaired persons. Archives of Ophthalmology, 116(2), 227-233.

Mangione, C., Phillips, R., Seddon, J., Lawrence, M., Cook, E., Dailey, R., \& Goldman, L. (1992). Development of the activities of daily vision scale: A measure of visual functional status. Medical Care, 30(12), 11111126.

Meereis, E., Lemos, L., Pranke, G., Alves, R., Teixeira, C., \& Mota, C. (2011). Deficiência visual: Uma revisão focada no equilíbrio postural, desenvolvimento psicomotor e intervenções. Revista Brasileira de Ciência e Movimento, 19(1), 108-113.

Messa, A., Nakanami, C., \& Lopes, M. (2012). Qualidade de vida de crianças com deficiência visual atendidas em ambulatório de estimulação visual precoce. Arquivos Brasileiros de Oftalmologia, 75(4), 239-242.

Moreno, B., \& Ximénez, C. (1996). Evaluación de la calidad de vida. In G. Buela-Casal, V. Caballo, \& J. Sierra (Eds.), Manual de evaluación en psicología clínica y de la salud (pp. 1045-1065). Madrid: Siglo XXI.

Murtagh, K., \& Hubert, H. (2004). Gender differences in physical disability among an elderly cohort. American Journal of Public Health, 94, 14061411.

Organização Mundial da Saúde (1994). WHOQOL Group. Development of the WHOQOL: Rationale and current status. Intellectual Journal of Mental Health, 23, 24-56. 
Organização Mundial da Saúde (1995). WHOQOL Group. The World Health Organization Quality of Life Assessment (WHOQOL): Position paper from the World Health Organization. Social Science \& Medicine, 41, 1403-1409.

Organização Mundial da Saúde (1998). WHOQOL Group. The World Health Organization Quality of Life Assessment (WHOQOL): Development and general psychometric properties. Social Science \& Medicine, 46, 1569-1585.

Pereira, É., Teixeira, C., \& Santos, A. (2012). Qualidade de vida: Abordagens, conceitos e avaliação. Revista Brasileira de Educação Física e Desporto, 2, 241-250.

Phillips, D. (2006). Quality of life: Concept, policy and practice. London: Routledge.

Piteira, A. (2000). Percepções de qualidade de vida de jovens com necessidades especiais (Dissertação de mestrado não publicada). Universidade Técnica de Lisboa, Lisboa.

Raphael, D. (1996). Eleven debates concerning its measurement. In R. Renwick \& M. Nagler (Eds.), Quality of life in health promotion and rehabilitation: Conceptual approaches, issues and applications (pp. 146165). Thousand Oaks, C A: SAge.

Raphael, D., Brown, I., \& Renwick, R. (1996). Studying the life's of persons with developmental disabilities: Methodological lessons from the quality of life project. Toronto: Quality of Life Research Unit/Centre for Health Promotion.

Rapley, M. (2003). Quality of life research: A critical introduction. London: Sage Publications.

Renwick, R., \& Brown, I. (1996). The center for health promotion's conceptual approach to quality of life. In R. Renwick, I. Brown, \& M. Nagler (Eds.), Quality of life in health promotion and rehabilitation: Conceptual approaches, issues and applications (pp. 75-86). Thousand Oaks: Sage.

Ribeiro, J. (1994). A importância da qualidade de vida para a psicologia da saúde. Análise Psicológica, 2(3), 179-191.

Ribeiro, J. (1997). A promoção da saúde e da qualidade de vida em pessoas com doenças crónicas. In J. Ribeiro (Ed.), Actas do 2..$^{\circ}$ congresso nacional de psicologia da saúde (pp. 253-282). Braga: Instituto Superior de Psicologia Aplicada.

Rosser, R. (1997). A health index and output measure. In S. Stewart \& R. Rosser (Eds.) Quality of life: Assessment and application (pp. 79-98). Lancaster: MTP. 
Ruta, D., Garratt, A., \& Leng, M. (1994). A new approach to the measurement of quality of life: The patient-generated Index. Medical Care, 32(11), 1109-1126.

Schalock, R. (1996). Reconsidering the conceptualization and measurement of quality of life. In R. Schalock (Ed.), Quality of life: Conceptualization and measurement (pp. 123-139). Washington, DC: American Association on Mental Retardation.

Schalock, R. (2004). Quality of life from a motivational perspective. International Review of Research in Mental Retardation, 28, 303-319.

Schalock, R., Brown, I., Brown, R., Cummins, R., Felce, D., Matikka, L., et al. (2002). Conceptualization, measurement, and application of quality of life for persons with intellectual disabilities: Report of an international panel of experts. Mental Retardation, 40, 457-470.

Schalock, R., Gardner, J., \& Bradley, V. (2007). Quality of life across individuals, organizations, systems, and the community. Washington, DC: American Association on Intellectual and Developmental Disabilities.

Schalock, R., \& Verdugo, M. (2002). Handbook on quality of life for human services practitioners. Washington, DC: American Association on Mental Retardation.

Seidl, E., \& Zannon, C. (2004). Qualidade de vida e saúde: Aspectos conceituais e metodológicos. Caderno de Saúde Pública, 20(2), 580-588.

Serra, A., Canavarro, M., Simões, M., Pereira, M., Gameiro, S., Quartilho, M., et al. (2006). Estudos psicométricos do instrumento de avaliação da qualidade de vida da Organização Mundial de Saúde (WHOQOL-Bref) para português de Portugal. Psiquiatria Clínica, 27, 41-49.

Shin, D., \& Johnson, D. (1978). Avowed happiness as an overall assessment of the quality of life. Social and Indicators Research, 5, 475-492.

Skevington, S., Lotfy, M., \& O'Connell, K. (2004). The World Health Organization's WHOQLBREF quality of life assessment: Psychometric properties and results of the international field trial. Quality of Life Research, 13, 299-310.

Sloane, M., Ball, K., Owsley, C., Bruni, J., \& Roenker, D. (1992). The visual activities questionnaire: Developing an instrument for assessing problems in everyday visual tasks. Technical Digest Series of the Optical Society of America, 1, 26-29.

Steinberg, E., Tielsch, J., Schein, O., Javitt, J., Sharkey, P., Cassard, S., \& Sommer, A. (1994). The VF-14: An index of functional impairment in patients with cataract. Archives of Ophthalmology, 112(5), 630-638. 
Veiga, C., Silva, C., Domingues, I., Saragoça, J., \& Fernandes, L. (2014). Inclusão profissional e qualidade de vida. Vila Nova de Famalicão: FORMEM.

Verdugo, M., Caballo, C., Peláez, A., \& Prieto, G. (2000). Calidad de vida en personas con deficiencia visual: Informe final de investigación. Salamanca: Instituto Universitario de Integración en la Comunidad, Universidad de Salamanca.

Verdugo, M., Schalock, R., Keith, K., \& Stancliffe, R. (2005). Quality of life and its measurement: Important principles and guidelines. Journal of Intellectual Disability Research, 49(10), 707-717.

Wray, L., \& Blaum, C. (2001). Explaining the role of sex on disability: A population based study. The Gerontologist, 41, 499-510. 